\title{
Technè
}

La science au service de l'histoire de l'art et de la préservation des biens culturels

44 | 2016

Archives de l'humanité : les restes humains patrimonialisés

\section{Analyses organiques de baumes de momies provenant du site d'Antinoé}

Organic analyses of mummy balms from the Antinoé site

Juliette Langlois et Sandrine Pagès-Camagna

\section{(2) OpenEdition}

1 Journals

Édition électronique

URL : http://journals.openedition.org/techne/1034

DOI : 10.4000/techne.1034

ISSN : 2534-5168

Éditeur

C2RMF

Édition imprimée

Date de publication : 1 novembre 2016

Pagination : 55-59

ISBN : 978-2-7118-6339-6

ISSN : 1254-7867

Référence électronique

Juliette Langlois et Sandrine Pagès-Camagna, « Analyses organiques de baumes de momies

provenant du site d'Antinoé », Technè [En ligne], 44 | 2016, mis en ligne le 19 décembre 2019, consulté le 28 juillet 2020. URL : http://journals.openedition.org/techne/1034 ; DOI : https://doi.org/10.4000/ techne. 1034

\section{(9) $(\triangle \Theta \Theta$}

La revue Technè. La science au service de l'histoire de l'art et de la préservation des biens culturels est mise à disposition selon les termes de la Licence Creative Commons Attribution - Pas d'Utilisation Commerciale - Pas de Modification 4.0 International. 
Juliette Langlois

Sandrine Pagès-Camagna

\section{Analyses organiques de baumes de momies provenant du site d'Antinoé}

Organic analyses of mummy balms

from the Antinoé site
Résumé. L'étude des restes humains patrimonialisés, et plus particulièrement des momies égyptiennes, met en œuvre de multiples techniques scientifiques, telles que la radiographie et la tomographie X, la datation au carbone 14, la paléo-entomologie, la paléobotanique, la paléo-anthropologie, la paléoanatomopathologie, l'étude des textiles et de la polychromie ou encore l'analyse des substances naturelles utilisées pour l'embaumement. Pour l'analyse des substances naturelles, la chromatographie en phase gazeuse couplée à la spectrométrie de masse (GC/MS) ne nécessite qu'une très faible quantité

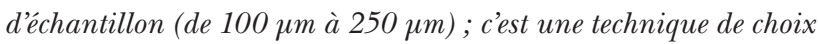
pour l'identification des résines végétales, des cires d'abeille et des corps gras, souvent présents dans les matériaux d'embaumement.

Mots-clés. Baumes, momies, substances végétales, analyses chromatographiques.
Abstract. The study of human remains that have acquired heritage status, Egyptian mummies in particular, involves several scientific techniques, such as X-rays, computed tomography, carbon dating, paleoentomology, paleobotany, paleoanthropology, paleopathology, the study of textiles and polychromy, as well as the analysis of natural substances used in embalming. When analysing natural substances, gas chromatography, coupled with mass spectrometry (GC-MS), requires only a minute sample (100 $\mu \mathrm{m}-250 \mu \mathrm{m})$. This is an excellent technique for identifying plant resins, beeswaxes and fatty substances, often present in embalming materials.

Keywords. Balms, mummies, plant substances, chromatographic analyses.
À l'occasion d'une vaste étude lancée en 2010-2011 par le Service du Récolement des Dépôts antiques et des Arts de l'Islam du musée du Louvre, ces techniques ont été mises en œuvre au C2RMF sur un corpus de momies provenant du site d'Antinoé (Égypte) et sur certaines ouvres faisant l'objet d'un projet de restauration, comme les momies du musée Joseph-Denais de Beaufort-en-Vallée (fig. 1). Les résultats des analyses chromatographiques des substances identifiées sur ce corpus sont présentés dans le tableau 1 page 56.

\section{Matières grasses}

La présence de matière grasse d'origine animale est mise en évidence par les acides myristique, palmitique, stéarique et oléique, qui sont associés à des acides gras dont la chaîne comporte un nombre impair d'atomes de carbone (acides gras linéaires en $\mathrm{C}_{15: 0}$ et $\mathrm{C}_{17: 0}$ ) et présente des ramifications (isomères iso et anteiso de la forme linéaire). Plusieurs isomères de position de l'acide gras $\mathrm{C}_{18: 1}$ et la présence éventuelle de cholestérol ou de ses dérivés en sont également les témoins.
Les matières grasses animales et végétales altérées étant principalement constituées d'acides gras libres identiques, seuls des composés secondaires, comme les diacides ou les acides gras à nombre impair d'atomes de carbone, permettent d'orienter la nature de la substance grasse présente. Dans le cas d'un mélange de graisse animale et végétale altérée, il reste très difficile, au seul moyen de la GC/MS, d'évaluer la proportion et la nature exactes de l'un et de l'autre. Les résultats résumés dans le tableau 1 concernent donc les matières grasses identifiées de façon prépondérante. Pour certains profils, les éléments identifiés sont insuffisants pour trancher entre matière grasse animale ou végétale ; on mentionne alors le terme de matière grasse au sens large. La présence d'une autre source de lipides que celle identifiée majoritairement est difficile à diagnostiquer pour les mêmes raisons.

C'est pourquoi, lors de l'analyse des baumes de momification, la nature des matières grasses animales est souvent difficile à déterminer avec certitude. Par ailleurs à cette période, elles peuvent provenir soit des baumes de momification, soit des restes humains eux-mêmes.

Juliette Langlois, assistant ingénieur, C2RMF (juliette.langlois@culture.gouv.fr). Sandrine Pagès-Camagna, ingénieur de recherches, C2RMF, département Recherche (sandrine.pages@culture.gouv.fr). 
Tableau 1. Substances naturelles identifiées sur le corpus des momies d'Antinoé étudiées au C2RMF

\begin{tabular}{|c|c|c|c|c|}
\hline Musée & $\mathbf{N}^{\circ}$ inv & Période & Localisation du prélèvement & Matériaux \\
\hline \multirow[t]{3}{*}{ Musée de l'Homme, Paris } & \multirow[t]{3}{*}{23313} & \multirow[t]{3}{*}{$\begin{array}{l}\text { Antinoé - } \\
\text { Copte }\end{array}$} & cheveux & $\begin{array}{l}\text { matière grasse }+ \text { traces de résine } \\
\text { de type Pinus sp. }\end{array}$ \\
\hline & & & textile végétal du bonnet & $\begin{array}{l}\text { matière grasse + résine de type } \\
\text { Pinus sp. }\end{array}$ \\
\hline & & & baume bras droit & matière grasse \\
\hline \multirow[t]{4}{*}{$\begin{array}{l}\text { Musée des Beaux-Arts, } \\
\text { Dunkerque }\end{array}$} & \multirow[t]{4}{*}{1972.00 .151} & \multirow[t]{4}{*}{$\begin{array}{l}\text { Antinoé - } \\
\text { Copte }\end{array}$} & textile végétal au niveau de la tête & $\begin{array}{l}\text { matière grasse + traces de résine } \\
\text { de type Pinus sp. }\end{array}$ \\
\hline & & & textile végétal entre les deux pieds & $\begin{array}{l}\text { matière grasse végétale (présence } \\
\text { des diacides de } \mathrm{C}_{6} \text { à } \mathrm{C}_{13} \text {, d'acide } \\
\text { 13,14-dihydroxydocosanoique, } \\
\text { marqueurs des huiles de graines } \\
\text { de Brassicaceae } \mathrm{sp} . \text { ) + résine } \\
\text { de type Pinus sp. }\end{array}$ \\
\hline & & & sous les côtes au niveau du bras gauche & $\begin{array}{l}\text { matière grasse }+ \text { traces de résine } \\
\text { de type Pinus sp. }\end{array}$ \\
\hline & & & cheveux & $\begin{array}{l}\text { matière grasse + résine de type } \\
\text { Pinus sp. }\end{array}$ \\
\hline $\begin{array}{l}\text { Musée d'art et d'archéologie } \\
\text { du Périgord, Périgueux }\end{array}$ & 4026 & $\begin{array}{l}\text { Antinoé - } \\
\text { Copte }\end{array}$ & textile végétal & $\begin{array}{l}\text { résine de type Pinus sp. oxydée } \\
+ \text { traces de matière grasse }\end{array}$ \\
\hline $\begin{array}{l}\text { Musée d'anatomie, } \\
\text { Faculté de Médecine, Lyon }\end{array}$ & 1448 & $\begin{array}{l}\text { Antinoé - } \\
\text { Copte }\end{array}$ & cheveux & matière grasse animale \\
\hline Musée des Confluences, Lyon & 90002978 & $\begin{array}{l}\text { Antinoé - } \\
\text { Copte }\end{array}$ & cheveux & matière grasse animale \\
\hline Musée des Confluences, Lyon & $90002977 \mathrm{~A}$ & $\begin{array}{l}\text { Antinoé - } \\
\text { Copte }\end{array}$ & cheveux & $\begin{array}{l}\text { matière grasse végétale } \\
\text { (présence des diacides } \\
\text { de } \mathrm{C}_{6} \text { à } \mathrm{C}_{13} \text {, d'acide } \\
\text { 13,14-dihydroxydocosanoique, } \\
\text { marqueurs des huiles de graines } \\
\text { de Brassicaceae } \text { sp.) + traces } \\
\text { de résine de type Pinus sp. }\end{array}$ \\
\hline Musée des Confluences, Lyon & 90002982 & $\begin{array}{l}\text { Antinoé - } \\
\text { Copte }\end{array}$ & textile végétal près du pied & matière grasse \\
\hline $\begin{array}{l}\text { Musée d'anatomie, Faculté } \\
\text { de Médecine, Montpellier }\end{array}$ & $\mathrm{E}$ & $\begin{array}{l}\text { Antinoé - } \\
\text { Copte }\end{array}$ & cheveux & matière grasse animale \\
\hline Musée des Beaux-Arts, Lille & 2 & $\begin{array}{l}\text { Antinoé - } \\
\text { Copte }\end{array}$ & cheveux & pas de signal significatif \\
\hline \multirow{2}{*}{$\begin{array}{l}\text { Muséum national d'histoire } \\
\text { naturelle, Nantes }\end{array}$} & \multirow[t]{2}{*}{ MHNN.E226 } & \multirow{2}{*}{$\begin{array}{l}\text { Antinoé - } \\
\text { Copte }\end{array}$} & baume sur textile au niveau de la hanche & gomme d'Acacia nilotica \\
\hline & & & textile au niveau de la hanche & matière grasse \\
\hline \multirow{6}{*}{$\begin{array}{l}\text { Musée Joseph-Denais, } \\
\text { Beaufort-en-Vallée }\end{array}$} & \multirow[t]{6}{*}{ BF3644 } & \multirow{6}{*}{$\begin{array}{l}\text { Antinoé } \\
\text { - Copte } \\
\text { Momie } \\
\text { d'une } \\
\text { "prêtresse } \\
\text { isiaque " }\end{array}$} & textile végétal sur le genou droit & matière grasse \\
\hline & & & cheveux & $\begin{array}{l}\text { matière grasse végétale (dont } \\
\text { AGC22:1 = Huile de graine de } \\
\text { Brassicaceae sp.) + traces de résine } \\
\text { de type Pinus sp. }\end{array}$ \\
\hline & & & baume sur le front & matière grasse animale \\
\hline & & & main droite & matière grasse \\
\hline & & & bloc de sable aggloméré entre les pieds & $\begin{array}{l}\text { matière grasse végétale } \\
\text { (présence des diacides } \\
\text { de } \mathrm{C}_{6} \text { à } \mathrm{C}_{14} \text {, d'acide } \\
\text { 13,14-dihydroxydocosanoique } \\
\text { et d'acide } \\
\text { 11,12-dihydroxyeicosanoique, } \\
\text { marqueurs des huiles de graines } \\
\text { de Brassicaceae } \text { sp.) + traces de } \\
\text { matière grasse animale }\end{array}$ \\
\hline & & & guirlande sur les cheveux & $\begin{array}{l}\text { matière grasse + traces de résine } \\
\text { de type Pinus sp. + traces } \\
\text { d'une résine tri-terpénique }\end{array}$ \\
\hline $\begin{array}{l}\text { Rouen, Musée départemental } \\
\text { des antiquités }\end{array}$ & AEg 410/1857.1 & Copte & $\begin{array}{l}\text { momie de femme recouverte de tissus } \\
\text { coptes }\end{array}$ & cire d'abeille altérée \\
\hline
\end{tabular}


Fig. 1. Substances naturelles prélevées sur une momie copte du musée Joseph-Denais (BF3644). (c) C2RMF/A. Chauvet.

\section{Le cas particulier des huiles de la famille des Brassicaceae}

La présence d'acides myristique, palmitique, stéarique et oléique est associée, dans le cas des huiles végétales siccatives (huile de lin, œeillette, noix...), à une série de diacides à chaîne linéaire allant habituellement de 6 à 10 atomes de carbone, le diacide azélaïque comprenant 9 atomes de carbone étant majoritaire.

Ces diacides, considérés comme des biomarqueurs des huiles végétales siccatives lorsqu'ils sont relativement abondants par rapport à l'acide palmitique et stéarique, proviennent de la dégradation d'acides gras insaturés lors de processus d'oxydation, pouvant être favorisés par la lumière et la température, et aboutissant à un clivage de la double liaison. La position de la double liaison sur la chaîne hydrogénocarbonée de l'acide gras «précurseur » détermine la longueur de la chaîne du diacide provenant de sa dégradation.

Le cas des huiles provenant des espèces de la famille des Brassicaceae (anciennement nommées Cruciferae) est différent, et on observe alors des diacides dont la chaîne linéaire va de 6 à 13 atomes de carbone. Les acides gondoïque (acide 11-eicosenoique) et érucique (acide 13-docosenoique) sont en effet relativement abondants dans la composition des huiles provenant de graines de cette famille. Selon un processus analogue à celui décrit précédemment, les acides gondoïque et érucique aboutissent respectivement, lors de leur oxydation, aux diacides linéaires à 11 et 13 atomes de carbones.

Des traces d'acide 13,14-dihydroxydocosanoique provenant, selon un processus semblable, d'oxydation de la double liaison de son précurseur, l'acide érucique ((Z)-13-AG C22:1) ${ }^{1}$, peuvent parfois être décelées dans les échantillons à l'aide du couplage à la spectrométrie de masse. L'association de ces dérivés di-hydroxylés aux acides eicosanoique (AG C20:0), docosanoique (AG C22:0) et tétracosanoique (AG C24 :0), mieux conservés car ne comportant pas de double liaison, est caractéristique des huiles végétales issues des graines provenant des Brassicaceae.

La famille des Brassicaceae présente de nombreuses espèces, parmi lesquelles on trouve le radis (Raphanus sativus L.), le navet (Brassica rapa L.) et le colza (Brassica napus L.). On considère que l'emploi de l'huile issue des graines de radis est répandu en Égypte romaine ${ }^{1}$; cependant, les confusions possibles lors des traductions et interprétations des textes anciens comme ceux de Pline, ainsi que la faible quantité d'huile contenue dans les graines de radis ${ }^{2}$, incitent à évoquer, d'une façon plus large, les espèces de la famille des Brassicaceae pour origine de ces huiles. Ce type d'huile a été identifié à plusieurs reprises comme combustible dans des lampes à huiles issues de vestiges de céramique égyptienne d'époque romaine provenant du site d'Antinoé ${ }^{1}$ (V-VII siècle av. J.-C.) mais également dans des céramiques d'époque chrétienne sur le site de Qasr Ibrim en Nubie ${ }^{3}$.

Préciser le type exact de végétal employé à cette période devient délicat, d'autant que les restes archéologiques se présentent souvent sous la forme de graines d'environ $0,1 \mathrm{~cm}$ de long, ce qui rend leur identification précise délicate : des graines de la famille des Brassicaceae ont été retrouvées dans la tombe de Toutankhamon ${ }^{4}$ et l'on a retrouvé des graines de navet (Brassica rapa L.) à la période romaine ${ }^{5}$. 


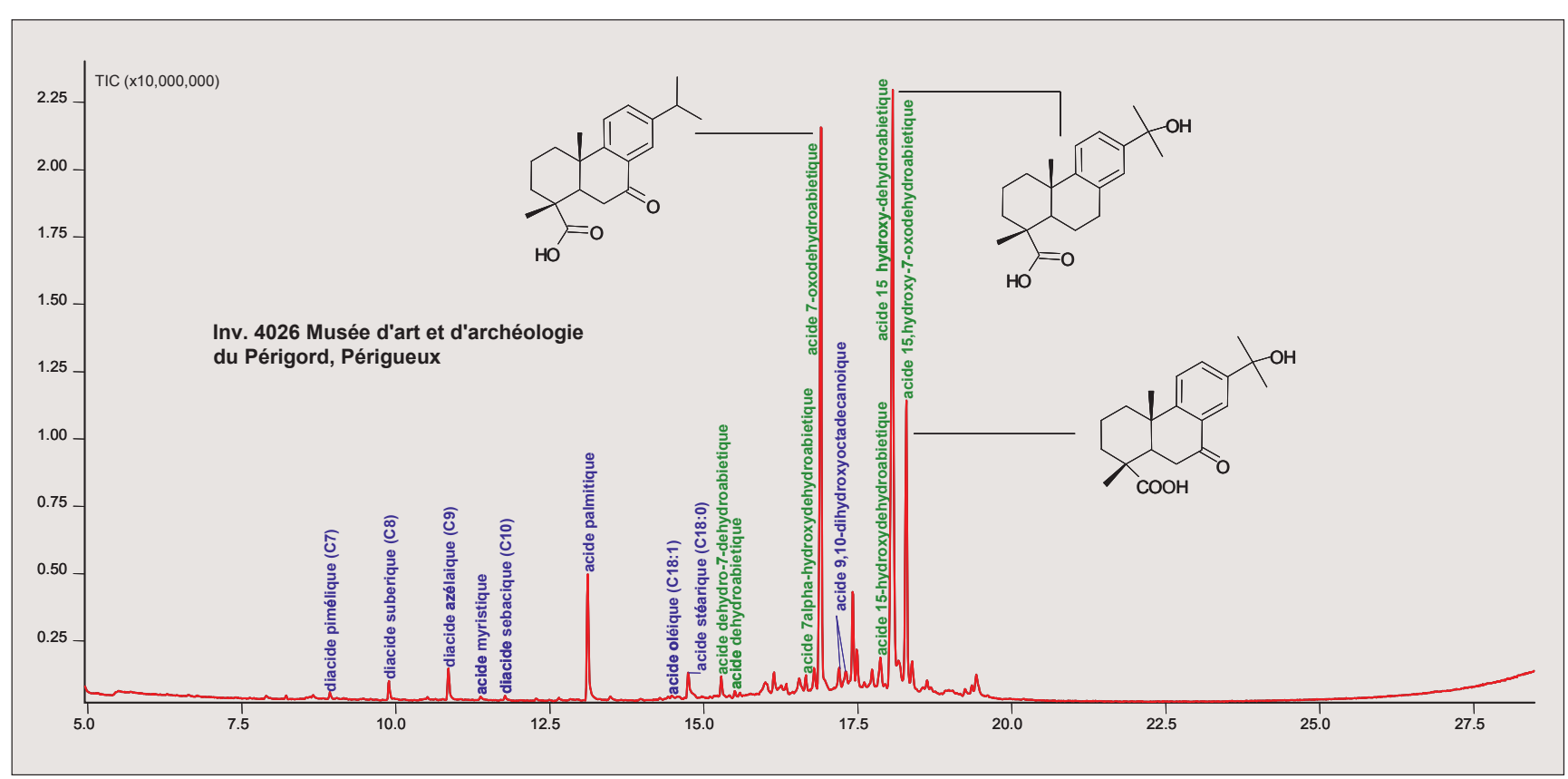

Fig. 2. Chromatogramme de la substance naturelle présente sur un textile végétal de la momie du musée d'Art et d'Archéologie du Périgord, Périgueux, analyse GC/MS-15m.

\section{Résine de Pinaceae sp.}

Les structures abiétanes (fig. 2) sont caractéristiques des résines naturelles provenant d'espèces de la famille des Pinacées (Pinaceae). Elles peuvent apparaîtrent relativement oxydées, par exemple lorsque les acides 7b (ou 7a)-hydroxydéhydroabiétique et 15-hydroxy-7oxodéhydroabiétique, sont présents. Dans certains cas, l'acide 15-hydroxy-dehydroabiétique partiellement méthylé (et non triméthylsilylé par le traitement de dérivation préalable à l'analyse) peut servir d'indicateur d'un chauffage de cette résine. En effet, cette méthylation intervient sur ces acides diterpéniques en raison du méthanol dégagé lors d'une combustion (Garnier, 2003) et non d'une oxydation naturelle.

\section{Cire d'abeille altérée}

Les caractéristiques les plus évidentes concernant l'identification d'une cire d'abeille sont la présence d'une série d'esters palmitiques à nombre pair d'atomes de carbone compris entre 40 et 50, ainsi que leur répartition avec l'ester à 46 atomes de carbone majoritaire. En plus de ces composés, la cire d'abeille contient une série de n-alcanes à nombre impair d'atomes de carbone, compris entre 23 et 33 , dont l'heptacosane est le composé majoritaire, et une série d'acides gras linéaires de 24 à 34 atomes de carbone.

En contexte égyptien, et ce quelle que soit la période, on observe fréquemment sur les profils, une altération partielle de la composition native de la cire d'abeille. Les alcanes et les acides gras linéaires présents à l'origine peuvent être très minoritaires, voire absents des matériaux étudiés. Une hydrolyse partielle des esters les plus légers (à 40 et 42 atomes de carbone) est parfois également visible. Ces phénomènes d'altération de la cire peuvent témoigner d'un processus de dégradation ou de migration en surface puis de sublimation de ces composés, compte tenu du contexte aride de leur conservation d'origine ${ }^{6}$.

\section{Gomme naturelle d'Acacia sp.}

Vérifier la présence éventuelle d'une gomme naturelle nécessite un protocole opératoire adapté, qui diffère de celui employé pour les lipides, cires ou résines : il s'agit en premier lieu d'hydrolyser les polysaccharides en monosaccharides par une méthanolyse acide. Puis, après neutralisation et évaporation, les fonctions alcools des monosaccharides sont dérivées par un réactif de triméthylsilylation afin de faciliter leur élution et leur séparation en chromatographie en phase gazeuse $^{7}$. Ce protocole permet de détecter la présence des monosaccharides constitutifs des polysaccharides que l'on trouve dans les gommes naturelles (gomme arabique, gomme de cerisier, gomme adragante...), ou dans l'amidon et la cellulose.

La gomme naturelle la plus fréquemment rencontrée en contexte égyptien est une gomme arabique, exsudat provenant de l'espèce Acacia sp., poussant au bord du Nil. Si son profil chromatographique est semblable à celui d'une gomme d'acacia d'Afrique de l'Ouest, l'Acacia sénegal sp. par exemple, l'absence du pic de rhamnose sous sa forme à pyranose est caractéristique des acacias de type tortilis, nilotica, nubica ou arabica ${ }^{8}$. 


\section{Conclusion sur le corpus des momies d'Antinoé}

Ces premiers résultats révèlent la prépondérance de l'utilisation de résine de la famille des Pinaceae. Aucune trace d'une autre résine végétale, comme la résine de pistachier (famille des Anacardiaceae, genre Pistacia), qui est parfois identifiée comme vernis sur des stèles ou des cercueils polychromes de la période pharaonique, n’a été décelée dans ce corpus.

Est-ce à mettre en relation avec l'organisation des circuits commerciaux de cette période, ces deux substances n'étant pas indigènes mais importées (île de Chios pour la résine de pistachier et pays du Moyen-Orient pour les résines Pinaceae), ou cela correspond-il à la valeur symbolique ou aux propriétés chimiques dans la conservation des corps de la résine de Pinaceae sp. par rapport à la résine de pistachier, dont on réserve l'usage à la polychromie d'objets décoratifs ?

L'utilisation de substances bitumineuses n'a pas non plus été mise en évidence lors de ces analyses, ce qui témoigne d'une modification des procédés d'embaumement à cette période plus récente par rapport à la période pharaonique où du bitume est parfois identifié.
En ce qui concerne les corps gras, il est souvent difficile d'en définir l'origine avec certitude, végétale ou animale, matériaux d'embaumement ou restes humains, hormis dans le cas des huiles de Brassicaceae sp. dont la signature est particulière. Cependant, il existe d'autres sources d'huiles végétales en Égypte : huile de balanos (Balanites aegyptiaca), huile de ben (Moringa peregrina ou Moringa aptera...). L'huile de raphanos (Brassicaceae sp.) est citée dans des papyri grecs provenant de la région du Fayoum, de période gréco-romaine, pour un usage médicinal sous forme d'onguent (Papyrus Amherst II, papyri Oxyrhynchus VI et XVI) ${ }^{9}$.

\section{Remerciements}

Ces analyses chromatographiques ont pu être entreprises lors de la campagne d'étude du corpus d'Antinoé en datation C14, publiée par ailleurs ${ }^{10}$; que Pascale Richardin, Nathalie Gandolfo et Noëlle Timbart en soient remerciées.
Notes

1. Lucas et Harris, 1962 ; Serpico et White, 2000.

2. Keimer, 1984 1998.

3. Copley et al., 2005 ; Regert et al.,

4. De Vartavan, 1990.

5. Serpico et White, 2000.

6. Regert et al., 2001.

7. Bleton et al., 1996 ; Méjanelle, 1996.

8. Anderson et al., 1966.

9. Lucas et Harris, 1962 ; Pline, XV, 7. 2013.

10. Cadot et al., 2013 ; Guérin et al.,

\section{Bibliographie}

Anderson D. M. W. and Karamalla K. A., 1966, "Studies on uronic acid materials. Part XII. The composition of Acacia Gum Exudates", Journal of the Chemical Society, Section C, p. 762.

Bleton J., Méjanelle P., Sansoulet J., Tchapla A, 1996, "Characterization of neutral sugars and uronic acids after methanolysis and trimethylsilylation for recognition of plant gums", Journal of Chromatography A 720: 27-49.

Cadot L., Coudert M., Dal Prà P., Gandolfo N., Langlois J., Richardin P., Timbart N., Weygand S., 2013, « Etude et restauration de deux momies du musée Joseph-Denais de Beaufort-en-Vallée ", Technè $\mathrm{n}^{\circ}$ 38, Science et conservation, p. 59-64.

Colombini M. P., Modugno F., Ribechini E., 2005, "Organic mass spectrometry in archaeology: evidence for Brassicaceae seed oil in Egyptian ceramic lamps", Journal of Mass Spectrometry 40: 890-898 ${ }^{11}$.

Colombini M. P., Giachi G., Modugno F., Ribechini E., 2004, "Characterisation of organic residues in pottery vessels of the Roman age from Antinoe (Egypt)", Microchemical Journal 79: 83-90.

Copley M. S., Bland H. A., Rose P., Horton M., Evershed R. P., 2005, "Gas chromatographic, mass spectrometric and stable carbon isotopic investigations of organic residues of plant oils and animal fats employed as illuminants in archaeological lamps from Egypt", The Analyst 130 (6): 860-871.

De Vartavan C., 1990, "Contaminated plantfoods from the tomb of Tutankhamun: a new interpretative system", Journal of Archaeological Science, 17: 473-494.

Keimer L., 1984, Die Gartenplanflanzen im alten Ägypten II. DAIK. ed. Germer, Mainz am Rhein.

Garnier N., 2003, "Structural analysis of organic materials preserved in ceramics from Antiquity: new developments in chromatography and mass spectrometry", Thèse de doctorat, Université Pierre-et-Marie-Curie, Paris VI. p. 222-239.

Guérin M.-L., Mainterot P., Brunet A., Langlois J., Gandolfo N., Richardin P., Cadot L., Timbart N., 2013, « Étude et restauration de la momie égyptienne du muséum de Nantes : exemple d'une collaboration pluridisciplinaire ", La Lettre de l'OCIM, n 148 , p. 12-20.

Lucas A., Harris J. R., 1962, Ancient Egyptian Materials and Industries, 4th edition,
Edward Arnold, London, p. 329

Méjanelle P., 1996, «Contribution à l'étude de substances organiques (gommes, résines et corps gras) mises en œuvre pour la réalisation d'objets d'art et d'archéologie : caractérisation par couplage chromatographie en phase gazeuse-spectrométrie de masse ", Thèse de doctorat, Université Paris VI.

Pline, XV, 7.

Regert M., Bland H. A., Dudd S. N., Van Bergen P. F., Evershed R. P., 1998, "Free and bound fatty acid oxidation products in archaeological ceramic vessels", Proceedings of the Royal Society of London, Series B: Biological Sciences B (265): 2027-2032.

Regert M., Colinart S., Degrand L. et Decavallas O., 2001, "Chemical alteration and use of beeswax through time: accelerated ageing tests and analysis of archaeological samples from various environmental contexts", Archaeometry, 43 (4), p. 549-569.

Serpico M., White, R., 2000, "Resins, amber and bitumen", in Ancient Egyptian Materials and Technology (Eds. Nicholson P. et Shaw I.), Cambridge University Press, Cambridge, p. 459.

Serpico M., White R., 2000, "Oil, fat and wax", in Ancient Egyptian Materials and Technology (Eds. Nicholson, P. \& Shaw, I.), Cambridge University Press, Cambridge, p. 390-429.

Serpico M., White R., 2001, “The use and identification of varnish on New Kingdom funerary equipment", in Colour and painting in ancient Egypt (Ed. Davies, W. V.), British Museum Press, London, p. 33-42. 